Voix et Images

volxetimages

\title{
Garnisons du Canada : Entre la mémoire et la promesse
}

\section{Naïm Kattan}

Volume 6, numéro 2, hiver 1981

Jean-Claude Germain

URI : https://id.erudit.org/iderudit/200265ar

DOI : https://doi.org/10.7202/200265ar

Aller au sommaire du numéro

\section{Éditeur(s)}

Les Presses de l'Université du Québec

\section{ISSN}

0318-9201 (imprimé)

1705-933X (numérique)

Découvrir la revue

\section{Citer cet article}

Kattan, N. (1981). Garnisons du Canada : Entre la mémoire et la promesse. Voix et Images, 6(2), 235-244. https://doi.org/10.7202/200265ar d'utilisation que vous pouvez consulter en ligne.

https://apropos.erudit.org/fr/usagers/politique-dutilisation/ 


\title{
Garnisons du Canada: Entre la mémoire et la promesse
}

\author{
par Naim Kattan
}

Peu importe si les apèlerins" du Mayflower ou les missionnaires de la Nouvelle-France ont obéi aux injonctions du départ plusieurs fois réitérées dans la Bible et auxquelles ont obéi Abraham, Jacob et Moïse.

A une ambassade qui l'exhorte à quitter la Huronie, Brébeuf dit: «Nous leur répondimes que nous n'étions pas venus en ces pays pour servir de truchement, ni sous espérance de nous $y$ enrichir ou de devenir un jour grand capitaine; mais que nous avions abandonné nos parents, nos moyens et toutes nos possessions et avions traversé la mer afin de leur venir enseigner la voie du salut au péril de nos vies.

Deux siècles plus tard, un poète canadien anglais: E.J.M. Pratt chante l'épopée de Brébeuf et de ses frères, évoque leur martyr qui est à la lois défaite et échec. Ainsi. Brébeuf représente une dimension de la mémoire de tous les Canadiens. C'est dire que ceux-ci acceptent que leur histoire ne commence pas comme une entreprise de conquête et une quête de richesse. Lès missionnaires ne sont pas partis d'une Europe d'oppression par désenchantement. Ils prétendaient sauver des âmes. Ils ne sont pas venus seuls. Les soldats et les trappeurs s'inscrivent eux aussi dans l'histoire mais dans l'imaginaire, ef l'imaginaire littéraire surtout, les soldats de Dieu occupent la place la plus haute, la place privilégiée.

Le Canada n'a donc pas été peuplé par des Européens malheureux qui cherchaient dans un nouveau départ à établir le royaume de Dieu dans la liberté. Aussi, l'espace nouveau n'impliquait ni séparation, ni cassure. Les nouveaux-venus se sont vite rendus compte que si cet espace pouvait assurer la durée dans le service de Dieu, il ne pouvait offrir la continuité dans la vie quotidienne. «Terre de Cain" a dit Jacques Cartier, car. cet espace sauvage interdisait l'expression d'une liberté à moins qu'elle ne fût synonyme de dissolution dans la nature et d'une disparition dans les éléments. II fallait donc composer avec une nature hostile et se défendre contre une population indigène qui, refusant d'étre dépossédée, était tout aussi hostile. Les voyageurs parcouraient le continent, établissaient postes et villages au centre et dans les Prairies. L'abondance n'était pas à la portée de la main et le grand espace 
sauvage ne se prêtait pas à une conquête facile ni à l'expression de la puissance de l'homme et de sa liberté. A la loi imposée par la nature a succédé la loi de l'homme. Plus tard, les marchands britanniques se sont trouvés aux prises avec des paysans britanniques eux aussi mais qui les ont précédés sur cette terre nouvelle. La loi et l'ordre britanniques ont régné après la Conquète et la Rébellion. Ce qui importe. ce n'est pas tant la source de la loi qu'elle fùt celle des Bourbons, des Eglises catholiques, presbrtériennes et anglicanes ou celle de la capitale de l'Empire et du Dominion, mais la reconnaissance d'une loi. l'acceptation de taire l'expression brute et directe d'un désir manifesté dans la spontanéité et l'immédiat. C'est le règne du prolongement d'une tradition, la durée, la naissance et l'élaboration lente d'une nouvelle tradition, si lente qu'elle est à peine perceptible, qu'elle se distingue à peine de celle. lointaine, qui lui a donné naissance et dont elle s'est séparée. Ce fut du moins le cas de la littérature. Les Relations des Jésuites étaient destinées à des lecteurs de la métropole et les récits de Susannah Moodie furent la réaction d'une Européenne affrontant l'espace nouveau.

Si aux Etats-Unis, à la suite des * pèlerins *, les immigrants désenchantés des vieilles cités d'oppression étaient partis à la poursuite d'une promesse, au Canada ils trouvaient, souvent sans le chercher, le prolongement d'une Europe qui imposait son pouvoir et sa loi. Aux Etats-Unis, la liberté s'exprimait par les hors-la-loi qui dévastaient le continent, faisant taire l'Indien et mettant en marche les moulins d'argent et de rève. Au Canada la liberté fut l'affranchissement d'un pouvoir d'outre-mer et l'acceptation de la loi que ce pouvoir a léguée en en faisant la base mème de l'expression du désir et de l'élaboration d'une tradition.

Face à une nature hostile, il fallait forger une loi, qui imposerait une discipline à la lutte et qui permettrait la protection d'une vie menacée. Or la loi d'outre-mer, eût-elle pour origine Londres ou Paris était lointaine. inadéquate, souvent irréconciliable avec les conditions dans lesquelles vivaient les nouveaux colonisateurs, trappeurs et coureurs de bois. De plus, elle favorisait les marchands qui débarquaient dans une terre déjà défrichée, dans une nature domestiquée durant des dizaines d'années d'effort, de courage et de misère. Protégés par le pouvoir lointain, ils récoltaient les fruits de générations de sacrifices. En plus d'être lointaine, la métropole apparaissait injuste. Pour les francophones, elle était de surcroît oublieuse et négligente. abandonnant ses enfants vaincus à la merci du nouveau pouvoir victorieux. La menace de l'hégémonie américaine a réuni les deux peuples dans leur opposition à la rupture avec la métropole, rupture qui apparaissait comme une transgression de la loi. Ils n'obéissaient pas par crainte mais par loyalisme, volontairement, et trouvaient la liberté dans la fidélité, le prolongement de la famille plutôt que dans la cassure.

La loi renaissait sous d'autres formes. Elle obéissait aux conditions matérielles, différentes d'une région à l'autre, à la mémoire déjà constituée des deux peuples et aux attentes de l'avènement d'un monde nouveau né d'une promesse non formulée. Ainsi, chaque Société mettait en place sa loi. C'est ce que Northrop Frye appelle l'esprit de garnison. Loi défensive protectrice 
qui aménageait les rapports d'une communauté fragile qui se sentait menacée par une nature cruelle. Au Canada français, c'est l'Église qui incarnait la garnison, qui érigeait ses clôtures et exprimait ses interdits et qui, en même temps, dispensait les assurances d'une sécurité et d'une consolation devant l'inévitable. La littérature est née d'abord d'une défense et illustration de l'esprit de garnison puis par un refus, une révolte contre sa rigidité mutilante et irrespirable. De Marie de l'Incarnation a Maria Chapdelaine, la garnison c'est la durée. la chaleur d'une communauté dont les membres se serrent les uns contre les autres au point de s'étoufler afin de trouver dans leur propre chaleur l'assurance d'une survie dans un espace glacial sans frontières. II fallait élever les frontières, les dessiner, les dresser pour se sentir en sécurité et aspirer à une liberté qu'octroie l'obéissance à la loi.

Dans la poursuite de l'aventure du nouveau monde, mémoire et promesse étaient indissolubles et ce n'est que par l'éclatement de la garnison qu'apparurent au grand jour leur fragilité et leur ambiguïté. Dans Maria Chapdelaine la mémoire est acceptation et la promesse attente. Et ni l'attente ni l'acceptation n'ont d'objet précis. Coupés de la France, les Canadiens français qui ont choisi la continuité devaient rapatrier la mémoire. D'abord la France elle-même, par la Révolution, a rompu sa propre continuité. II était possible dès lors, soit que l'aventure dans la mémoire prenne son point de départ en Amérique. soit qu'elle recoure à une mémoire faite de nostalgie et d'une affirmation que la métropole réelle n'est pas celle qui existe mais celle qui fut détruite, celle avec laquelle les révolutionnaires, usurpateurs de légitimité ont rompu.

Deux nationalismes, deux spiritualités, j'allais presque dire deux théologies. Dans Menaud maître draveur, Félix-Antoine Savard décrit le réel. Ici, la continuité est faite d'endurance et d'entêtement dans l'attente. Dans la Dalle-des-Morts, laventure du nouveau monde est héroïque, faite d'un investissement de l'espace non par volonté de conquête mais par affirmation et mise en ceuvre d'une volonté divine. Chez l'Abbé Groulx, la mémoire est une histoire et celle de la Nouvelle-France est un prolongement de celle de la France réelle, la France nón perturbée par la Révolution. La mémoire est rapatriée. Elle est dotée d'une direction, d'une idéologie sinon d'un séns. Dans cette entreprise, la promesse est ensevelie dans les exigences du devoir. de la mission. Cette continuité inter dit la résurgence du désir. Le devoir impose la discipline et la noblesse de la mission sa mise entre parenthese. Oans /Appel de la race. le mariage el l'amour eux-mêmes, sont soumis à ces exigences.

L'esprit de la garnison implique la cohésion de la communauté. Toute influence extérieure la contamine, dégrade son attente. Il est illusoire d'oublier le monde extérieur car il est là et c'est contre son hostilité que la garnison s'est constituée. Aussi, dans Maria Chapdelaine, lappel au large est rejeté au profit de l'attente et dans l'Appel de la race l'intrusion de l'étranger fait ressortir l'importance de la cohésion de la communauté de garnison. Et cet appel au large, ce vaste monde qui entoure la garnison persiste et exerce la séduction. II se transforme en rêve. Le monde est entrevu à travers la clôture et l'étranger n'est admis dans l'enceinte qu'à condition qu'il soit reconnu comme étranger 
qui dérange la continuité. mais ce n'est que pour renforcer et réaffirmer la cohésion. C'est le Survenant qui rôde, qui fait rêver, qui peut briser les cceurs mais qui demeure en marge puisqu'il n'est que de passage. La persistance du Survenant, de l'homme de passage est, comme dans Maria Chapdelaine une reconnaissance a la fois de la fragilité de la communauté et de son importance. Le Survenant est aussi l'autre possible. La porte est ouverte. II suffirait d'avoir la force. I'audace et le courage pour y passer. Mais c'est accepter alors l'autre versant de l'aventure américaine, celle des États-Unis. Du moins, c'est ainsi que l'entreprise est percue. Quitter l'enceinte, cela veut dire se trouver seul, se découvrir démuni, coupé de sa mémoire.

Au Canada anglais, la mémoire est tout autant une dimension de l'aventure et elle est tout autant brisée et ambigüe. Si la loi est britannique. l'ensemble des communautés ne l'est pas. Pour certains, la fidélité à la mémoire est un loyalisme à la mémoire des autres. Ainsi, le héros de l'Ouest est le Mountie et non pas le cowboy, cet homme libre qui est parfois un hors-la-loi. Le Mountie, c'est l'ordre qui prend sa source dans la loi britannique. C'est là la forme que prend le loyalisme dans son investissement du grand espace nouveau. Cet espace n'est pas le domaine où l'homme s'éprouve, exerce sa force, affirme sa puissance et découvre sa liberté. C'est le territoire où ii entrevoit ses limites et, dans les frontières qu'il trace, érige les murs protecteurs. La Loi de Dieu est la sécurité suprême. Loi que chaque communauté redéfinit, récrit à son propre bénéfice. Et sous l'empreinte d'un double empire, celui de Dieu et celui de la métropole européenne, chaque communauté sécrète ses lois. Le châtiment du transgresseur est sévère et rigide même quand il n'est pas énoncé. Au Canada français comme au Canada anglais, la cohésion des communautés est implacable. Pour certains, elle est étouffante mème quand elle dispense protection et sécurité. Des ceuvres parmi les plus marquantes, les plus significatives evoquent une diversité de tentatives de libération. Dans Poussière sur la ville, Langevin décrit la monotonie débilitante du quotidien où l'individu se sent absorbé, perdu comme personne. Dans Le temps des hommes, l'individu, en l'occurrence un prêtre qui cache son identité accepte la loi de Dieu, se voue à son service mais sent sa parole perdue. If cherche un nouveau départ dans l'espace mais ne trouve que l'échec et la mort. Dans I'Élan d'Amérique, cet individu perdu dans l'espace renaît de ses cendres. Il aspire au rêve américain, a la promesse dont il fut privé mais ne découvre que pacotille et vulgarité et son évasion dans l'espace n'est qu'une marche vers la mort.

Même quand le refus est naïf et la révolte juvénile comme dans Le libraire de Bessette, l'échec n'est pas moins outrecuisant. Car les enfants de Dieu sont mal nés. Les personnages de Marie-Claire Blais soutfrent d'une déformation de l'âme. La parole de Dieu qu'ils ne cessent d'entendre répéter ne les atteint pas et la débilité de l'âme atteint d'abord le corps, le réduit avant de le vouer à la mort.

A l'autre bout du continent on retrouve les mèmes petites villes perdues dans l'espace chez. Sinclair Ross et Sheila Watson. Dans As for me and my house nous avons encore affaire à un homme qui s'est voué à Dieu, comme 
malgré lui, par une sorte d'ironie, c'est-à-dire en meltant une distance, non par rapport à Dieu, puisqu'il ne croit pas, en dépit de lous les sermons qu'il prononce tous les dimanches à l'église. Une distance par repport à lui-même. Son amour ne rejoint pas le désir. II est frappé d'une incapacité de vivre qui se présente comme une stérilité dans son rapport aux autres et à lui-même. II ne peut être artiste qu'en secret, en pure perte. Cette même incapacité de vivre, cette impossibilité d'éliminer la distance qui sépare l'être de lui-même on les retrouve dans The Double Hook. Mais là, la révolte est plus viscérale et le relus plus puissant. La liberration n'est pas plus proche pour autant. C'est le même échec. la même route qui mène à la violence, à la lolie et au silence. Dans The Stone Angel Margaret Laurence a fait l'anatomie de la communauté de garnison. Hagar, et le rappel biblique ne me semble pas fortuit, ne peut quitter le désert où elle se trouve abandonnée qu'en reformulant la loi d'une communauté a laquelfe elle ne fut pas conviée. Sa rigidité envers la loi, son avarice d'âme représentent son droit d'entrée, sa carte d'adhésion à une communauté qu'elle constitue sans s'en rendre compte, en la vidant de toute vie. Le prix à payer est lourd: une vie sans amour, le désir vidé.

Dans The Fire Dwellers, le déplacement ne permet que le parcours de la surface de l'espace, et le recours à l'aventure individuelle fait de la vie privée une armure. II faudrait formuler alors les règles du jeu et, face à une société gagnée par l'avidité et l'insatisfaction, hommes et femmes se sentent perdus, incapables de trouver en eux-mêmes des sources nouvelles d'une reprise de la loi. La mémoire s'éloigne et se dissout dans un quotidien pesant. encombrant, dont la texture est la vacuité, l'inutilité et par conséquent l'incertitude. Aussi, faut-il, avant de reformuler la loi, retrouver la mémoire et pour cela il est nécessaire non pas de retourner en arrière mais d'aller dans le présent, au fond de soi, de fouiller dans les replis de l'âme et de l'esprit, les vestiges d'une vie autre et dans le passé, ce qui persiste et alimente un quotidien dépourvu de sens. II faut, en d'autres termes, être des sourciers (diviners). Dans ce roman, The Diviners, nous sommes en présence de personnages en quête de mémoire. Ce n'est pas tant l'origine raciale ou familiale qui est ensevelie dans le brouillard et lincertitude que la mémoire culturelle. Ils sont métissés, abandonnés par le père. Ils sont tous à la recherche de sources. Est-ce l'indigène indien métissé de francophone qui est le fondateur de l'Ouest ou bien est-ce l'immigrant écossais?

Morag part en Grande-Bretagne, le pays d'origine et ne se reconnait pas dans l'amant écossais auquel elle réclame autant une révélation des sources que l'amour. La source n'est ni dans l'origine proche ou lointaine, hi dans l'espace mais dans le lieu quion aménage tant bien que mal dans l'espace sauvage. A tout moment, on risque de se perdre dans l'anonymat du quotidien. La tentation est grande alors de s'accrocher à une abstraction que l'on érige comme definition mais qui n'est qu'un leurre auquel on s'attache pour que l'immensité glaciale n'ait pas le dessus.

Et c'est l'attente, une longue, une interminable attente. II $y$ a certes l'espoir d'une promesse mais le Canadien anglophone ou francophone sait qu'il n'a pas l'énergie pour aller au devant de la promesse. Sa démarche n'est pas 
celle du conquérant ni celle de l'abandon. II ne se résigne pas. Ses armes sont l'endurance et la patience. Une sagesse primaire dicte sa conduite. II n'a pas les moyens de la révolte. Pour endurer sans se résigner, sans perdre tout ressort, il lui reste le loisir de se plaindre. Les pièces de Marcel Dubé sont une longue plainte, un soupir ininterompu. Quand les personnages sont pauvres, c'est-a-dire quand l'adolescent appartient à la petite classe moyenne, if se plaint de la faiblesse de son père, de sa soumission à son patron, de la dureté du travail de la mère et quand le garçon ou la fille, et les pièces de Dubé abondent en adolescents, appartiennent a la bourgeoisie, ils se plaignent de la corruption du père, du désceuvrement de la mère, de la soif d'argent de tout le monde. La révolte aboutit au départ et le révolté ne se change pas lujmême ni ne cherche à changer la société. Il devient délinquant. C'est dire qu'il sait que toute révolte aboutit à la transgression et que refuser la loi est un péché. II peut se plaindre du malheur d'être né dans son pays, son milieu, sa famille.

D'autres. surtout les poètes. se demandent s'ils ne sont pas là par erreur, si le destin ne les a pas mal partagés. Sont-ils nés dans le bon pays, le bon milieu et la bonne famille? Le nouveau monde n'est pas le lieu où la promesse s'accomplira. L'attente est donc dérisoire et vaine. S'opposer? Clamer refus et révolte? Contre qui? Le mal atteint le fondement mème du destin. Le Nouveau Monde c'est l'exil. D'Archibald Lampman à Anne Hébert en passant par Charles Roberts le sentiment prévaut qu'un homme nouveau ne peut naitre dans cette terre sauvage. L'enfermement est sans issue puisqu'on est dépossédé de tout choix. Et si on s'était trompé de terre et de destin? L'Amérique n'est peut-être qu'une illusion et la civilisation est nulle part. Dans The Favorite Game, Leonard Cohen imagine une ville sans passé. II est donc possible qu'on se soit leurré quand on a appuyé toute l'existence de la nouvelle cité sur une mémoire. Les prètres de toutes les églises qui se sont succédés dans leur implantation sur cette terre et qui vivent côte à côte se sont peut-être trompés quand ils ont cru qu'ils étaient les porteurs de la parole de Dieu. Dans The Beautiful Losers. Cojen juxtapose l'aube de cette nouvelle terre avec son destin présent. Catherine Tekakwitha est une Indienne qui accepte la civilisation du Blanc, qui accepte son Dieu et sa parole, qui lui offre sa vie. Elle meurt et elle est déclarée sainte par l'Église. Édith, la maîtresse du narrateur est son incarnation d'aujourd'hui. Elle accepte elle aussi la civilisation teille qu'elle se présente, le plaisir du sexe et de la ville. Or, comme la sainte d'antan elle se heurte à un système d'enfermement. Les mots d'hier sont les machines d'aujourd'hui et son plaisir lui est dispensé par un vibrateur danois. II est possible que cette terre soit prisonnière de la mémoire qui l'a enfermée dans une loi qui vide le quotidien de sa substance et qui mécanise le plaisir et le sexe. Aveuglés par leur illusion. les Blancs n'ont pas vu la terre sauvage, $n$ 'ont pas accepté ce qui fait qu'elle soit nouvelle et qui est justement sa sauvagerie. Ils ont même dépossédé ses habitants de leur substance en les traitant paradoxalement de sauvages.

D'autres poètes, ceux de l'Hexagone, constatant la transformation des. religieux en théologiens et la parole en lois des hommes et en règles de la 
société. ont décidé de repartir à neuf, de retrouver linnocence du regard pour redécouvrir le pays. Ils se sont mis à en dénommer les lieux et les arbres. Pour échapper à l'exil il leur apparaissait possible et même nécessaire de reprendre l'entreprise dès le début, c'est-à-dire de réinvestir l'espace d'une conscience neuve sinon innocente, et finalement de prendre possession du champ sau. vage. Reste la mémoire, brisée, oblitérée par des leurres. des mensonges et des travestissements. Ils allaient la réinventer. Ils niallaient donc pas se contenter d'apprivoiser l'espace afin de le transformer en lieu. Ils allaient inventer un pays. Un pays à la recherche de sa mémoire et en quête de promesse. Mais d'abord le combat. Nous sommes loin de Jean de Brébeuf même s'il faut tracer la ligne de continuité. Au départ un constat. Ce pays est américain. Il portera un nom. Désormais les écrivains comme tous ceux qui le voudront, se nommeront Québécois. Nous sommes dans un territoire. L'espace a des frontières mais pour les délimiter il faut peut-être voir d'abord où se situe le domaine. Godbout, Aquin promènent les lecteurs de l'Afrique à IEurope en passant par le continent américain mais il n'y a pas à se tromper celui qu'ils mettent en scène et à qui ils cherchent à s'adresser est d'ici. Ils entreprennent den dessiner un portrait ressemblant qui sera tour a tour sarcastique, caricatural, évasif et incertain. L'espace a toujours existé mais on ne l'a jamais vu qu'enfermé dans des lois et des mols. On était doté d'âme et d'esprit mais on les a barricadés pour les protéger. Et voici non seulement l'espace qui échappe mais le monde. Les personnages de Réjean Ducharme sont des descendants de Montcalm mais aussi de Christophe Colomb. Le Irancophone d'Amérique a toujours eu des difficultés avec les mots. A l'extérieur de la garnison ce qui était menacé c'était son verbe. II savait qu'il ne pouvait pas prendre la langue pour acquis. Pour lui donner la sécurité des pouvoirs établis, l'Etat et l'Eglise l'entermaient dans des mots qu'ils lui présentaient comme armures. Dès qu'il se met à les manipuler il les soigne, il s'en méfie et il en a peur. En saisit-il vraiment la nature? Le sens n'est-il pas en déroute, dévové, dégradé? Pour se défendre contre la hantise et l'enfermement il $s^{\circ}$ adonne au jeu. Ainsi il est à distance, il n'est pas mis en question et ces armures qui le barricadent tombent de leur piedestal. Its sont de petits objets sans utilité. des outils de jeu. II se libérera de leur emprise en les réduisant à des instruments de divertissement. Les mots résistent, sont irréductibles même s'ils ne sont que des instruments de jeu. L'Amérique est donc pour l'écrivain francophone, un langage, des mots qui lui échappent et qu'il cherche à saisir. à contrôler. Aussi, chez Ducharme le jeu de mots n'est qu'une manière d'explorer l'espace, de s'y situer, d'y trouver sa place. L'Amérique, de Montcalm à Christophe Colomb, de la fille de celui-ci à la juive de New York est une création verbale. Sil'investissement du territoire du continent $n$ 'a pas accompli la promesse, c'est que le sens n'est que dans les mots et c'est par eux qu'il laut commencer. Ces instruments denfermement, ces outils des lois rigides peuvent aussi être des objets joyeux, des moyens d'exploration d'une Amérique qui nous entoure mais qui est aussi en nous, une dimension et une invention de notre esprit. Au bout des mots et des jeux. Ducharme arrive à une conclusion provisoire, certes. l'Amérique n'est pas une promesse et on ne quitte pas le lieu de l'enfermement en faisant dans l'imaginaire le parcours de tout le 
continent. II revient au territoire et dans /'Hiver de Force retrouve l'exil et la dérision de tant de ses prédécesseurs et contemporains. Et si la promesse américaine s'arrètait au rève? Et si, nous étions privés mème du rêve ? Victor Lévy-Beaulieu cherchera non pas à refaire l'exploration de l'Amérique par son imaginaire, de l'intégrer à une entreprise distincte mais d'en faire le tour pour la calquer. If cherchera des réponses chez Kerouac et chez Melville et écrira un roman au titre transparent, Le Rêve québécois.

Toutefois le ressort réel de cet écrivain, c'est le recours à l'intime, à la fragilité de la mémoire, au grand-père, a l'absence d'affirmation, aux ambiguités de l'exil et à la redécouverte d'une traditionnelle consolation qui allait être oubliée sous des énonciations de refus et de revendications. Je veux dire la plainte. Et nous voici à nouveau dans l'enceinte de la garnison. Elle est moderne, aménagée pour l'époque du confort et de la technologie. Elle se présente avec le vocabulaire du jour mais nous nous retrouvons à l'intérieur d'un territoire où le réve américain est enterré sous le poids de sa technologie pesante. sans visage, accueillie avec empressement.

Cette technologie fait regimber les autres Canadiens, les anglophones. La communauté de langue ne fait qu'accentuer la fragilité de leurs traits distinctifs.

La mémoire est tellement brisée. ensevelie par la technologie de l'Amérique industrielle et la prépondérance d'une multitude de groupes d'origine non britannique dens certaines régions du pays et notamment dans les Prairies. Les immigrants ukrainiens, allemands et juifs qui ont peuplé l'Ouest. le Centre et qui ont transformé le visage des grandes villes, d'abord Montréal et Winnipeg, puis Toronto et Vancouver, ne savaient pas au départ qu'ils s'installaient dans le pays du prolongement, de la continuité plutôt que dans celui de la rupture. Ils l'ont découvert assez rapidement et l'ont accepté car la contre-partie c'était leur propre continuité. Ils pouvaient impunément non seulement exprimer leur attachement à leur mémoire mais en déployer la singularité et méme la présenter parfois comme modéle. Peut-ètre ne se rendaient-ils pas compte qu'ils acceptaient d'observer la loi plutôt que d'attendre l'avènement d'un monde nouveau, la renaissance de l'homme et la réalisation de la promesse.

Je voudrais signaler le cas le plus frappant, celui des écrivains juifs. Citons d'abord quelques titres: Seize the day. A New Life. Professor of Desire. Les auteurs, on l'aura deviné ne sont pas Canadiens puisqu'il s'agit de Bellow. de Malamud et de Philip Roth. Citons maintenant d'autres titres. The Second Scroll. The Son of a Smaller Hero. The Sacrifice. J'ai cité a dessein ces trois romans de A.M. Klein, Mordecai Richler et Adele Wiseman. Dans les trois romans, les rouleaux de la loi jouent le rôle principal. Pour Klein. l'Amérique ne peut se fonder que sur la loi mais il faudra la récrire, rédiger un deuxième rouleau en fidélité, en concordance avec le premier. Il est curieux que dans les deux romans de Richler et de Wiseman, les rouleaux brûlent et on risque sa vie, on la sacrifie pour les sauver du feu. Alors que les écrivains américains parlent d'aventure, de nouvelle vie et de désir, leurs contemporains canadiens 
s'attachent aux rouleaux de la loi et sont hantés par leur disparition possible. Ainsi si les écrivains juifs américains acceptent et participent à l'expression du rêve américain, ceux du Canada s'inquiètent de la sauvegarde de la loi. $S$ 'ils se distinguent par leur mémoire, les Canadiens quels qu'ils soient, partagent le souci de la continuité et la peur de la rupture.

A cette crainte vient s'ajouter la peur de la perte de la mémoire. Pire encore, on ne parvient plus à discerner la substance de cette mémoire et sa nature. Son profil se perd dans l'incertitude. Dans Lament for a Nation, George Grant, sous le couvert d'une lamentation pousse un ultime cri, un cri de colère et d'alarme, un cri désespéré. Son point de repère n'est point l'Amérique car elle a préféré les promesses à la promesse. la quantité á la qualité, le confort aux valeurs. Mais la Grande-Bretagne qu'il invoque n'existe plus et semble elle-même contaminée. sinon par le rêve du moins par la technologie américaine. En lait, cette lamentation ne peut être que lamentation même si elle tombe souvent dans la nostalgie. Curieusement c'est une femme d'une autre génération qui, à première vue, peut paraître se placer aux antipodes du philosophe qui prend sa releve. Paradoxalement au moment où les Canadiens francophones ne parlent plus de survie, trouvent la notion et le mot lui-même surannés et suspects, Margaret Alwood intitule son manifeste sur la mémoire canadienne Survival. Produit de l'Amérique, nourrie du rêve américain, Atwood comme tant d'Américains des dures années de la guerre du Vietnam, voit la promesse américaine dégradée et le rêve américain enseveli dans le sang sous un amas de cadavres. Contrairement aux Américains dont certains sont venus chercher refuge ici, elle peut avoir recours à un passé autre, et à travers la plainte d'avoir été réduit au rôle de victime, clamer l'innocence d'un peuple qui n'a pas eu a jouer les bourreaux. La Grande-Bretagne? Pas un mot. Sur le passé impérial? Mutisme. Tout a commencé ici et elle aussi à la suite des Québécois a recours au pays. Elle parcourt le territoire et dans son roman Faire Surface elle croit que la terre elle-même est saccagée, abimée par les prédateurs américains. Elle se rend compte, quelle dérision, que ceux qu elle prenait pour des Américains étaient, en fait, des Canadiens. C'est dire l'étendue du mal. Peut-on repartir à zéro? Se faire une mémoire, la tracer, la délimiter et au besoin l'inventer? Une mémoire canadienne. Ni britannique, ni américaine. Ensuite explorer sinon l'espace du moins le territoire. Et pour affronter sa sauvagerie, son hostilité, accepter la vie sauvage. Peut-être ainsi peut-on se refaire une innocence pour ensuite attendre. Dans Ille au Dragon. Jacques Godbout n'en est pas loin lui qui dénonce avec autant de force le prédateur américain. Chez lui, comme chez Atwood, il n'est pas question de conquérir l'espace pour affirmer la présence de l'homme encore moins sa puissance. L'un et l'autre remettent à l'ordre du jour une attitude canadienne traditionnelle. L'espace est hostile et il faut obéir a ses lois, se soumettre à ses conditions pour le rendre habilable. pour le transformer en lieu. Protéger le lieu de lappétit destructeur et insatiable des prédateurs. progéniture des anciens dévastateurs du continent, c'est se protéger soi-même pour survivre. On a besoin des murs et des boucliers, des lois et de la discipline pour se prémunir contre la menace. N'est-ce pas une nouvelle mouture de l'esprit de garnison? Les sauvages ont changé de visage mais ils ne sont pas moins 
menacants. Et la promesse? Peut-elle poindre dans la sauvagerie de l'espace au-delá de la mémoire et de la loi ?

Dans son ouvrage Butterfly on Rock, D.G. Jones consacre un chapitre à ce qu'il qualifie le problème de Job. Même si je ne suis pas d'accord sur sa présentation du personnage de Job, je ne peux que me rallier à son sentiment qu'il s'agit d'un personnage essentiel pour l'interprétation de la littérature canadienne. Job n'est pas à l'instar de Moise un chef qui conduit son peuple aux confins de la terre promise, ni a l'exemple de David et de Salomon un souverain qui détient un pouvoir, pouvoir qui s'appuie sur la puissance divine et qui ne peut donc s'exercer sans se nier, autrement que dans la justice. C'est un homme seul, qui frappé subitement par l'injustice, se refuse de l'attribuer à Dieu. II n'a qu'une arme: la patience. Il endure les conseils de ses amis tout autant que les calamités successives et ne perd pas confiance en Dieu. II reconnait Sa puissance et l'accepte. II attend. II n'est pas question d'un nouveau départ et aucune terre promise ne se profile à l'horizon. Endurer dans la confiance et attendre dans une infinie patience. La vie n'est un recommencement que dans la continuité, dans la durée sans éclat. Car, privé de tous les bienfaits du monde, la mémoire demeure vivante et c'est elle qui se renouvelle et assure la durée. N'est-ce pas une autre manière de vivre la promesse? Le miracle n'est pas dans la traversée des mers et l'atteinte du nouveau rivage mais dans la reprise du quotidien. II n'est pas besoin d'un séjour de quarante ans dans le désert pour attester les vertus de l'attente. L'héroisme peut être aussi dans une patience muette. Job avait une maison. Ses murs n'étaient défendus que par la soumission a la volonté divine. Le long séjour dans la garnison aura appris au Canadien, quelle que soit sa langue, que la promesse peut aussi être une simple maison, un lieu bâti dans la confiance et gagné de haute lutte à coups de patience. 